\title{
EFEITO DA COLHEDORA, VELOCIDADE E PONTO DE COLETA NA CONTAMINAÇÃO DE SEMENTES DE SOJA POR FUNGOS ${ }^{1}$
}

\author{
DELINEIDE P. GOMES ${ }^{2}$, ADRIANA Z. KRONKA ${ }^{3}$, LEANDRA M. BARROZO ${ }^{4}$, ROUVERSON P. DA SILVA ${ }^{5}$, \\ APOLYANA L. SOUZA ${ }^{6}$, BRENO M. S. E SILVA ${ }^{7}$, RITA DE C. PANIZZI ${ }^{8}$
}

\begin{abstract}
RESUMO - A pesquisa foi realizada com o objetivo de avaliar a qualidade sanitária de sementes de soja cv. Coodetec 217, colhidas por duas colhedoras operando em velocidades e pontos de coleta distintos. O delineamento foi inteiramente casualizado, no esquema fatorial $2 \times 2 \times 2$. Os tratamentos foram constituídos por duas máquinas Massey Ferguson (MF 2003 e MF 2004), duas velocidades (4 e $7 \mathrm{Km} \cdot \mathrm{h}^{1}$ ) e dois pontos de coleta (tanque graneleiro e saída do tubo de descarga). A sanidade foi avaliada pelo método do papel de filtro, com quatro repetições de 50 sementes. Houve interação significativa para os fatores colhedora e ponto de coleta para a incidência de Penicillium sp. e Fusarium sp., entre colhedora e velocidade para Aspergillus spp., e, entre velocidade e ponto de coleta para Penicillium sp.. A presença de fungos associados às sementes, principalmente, Cladosporium sp. e Fusarium sp., independe do tipo de colhedora. Uma menor contaminação fúngica é observada nas sementes coletadas na saída do tubo de descarga à $4 \mathrm{Km} . \mathrm{h}^{-1}$. Para Fusarium sp., Aspergillus spp., e Penicillium sp. existe uma dependência dos fatores sobre suas incidências nas sementes da cultivar analisada, sob as condições estudadas.
\end{abstract}

Termos indexados - Glycine max, colheita mecanizada, dano mecânico, patologia de sementes

\section{EFFECT OF THE HARVESTER, SPEED AND COLLECTION PLACE ON THE CONTAMINATION OF SOYBEAN SEEDS BY FUNGI}

\begin{abstract}
The objective of this study was to evaluate the health quality of soybean seeds $\mathrm{cv}$. Coodetec 217, in two harvesters operating at different speeds and in different collection places in the machine. The treatment consisted of two machines (Massey Ferguson, 2003 and Massey Ferguson, 2004), two speeds $\left(4 \mathrm{Km} \cdot \mathrm{h}^{-1}\right.$ and $7 \mathrm{Km} \cdot \mathrm{h}^{-1}$ ) and two collection places (grain tank and unloading exit). The analyses were realized at the Laboratory of Phytopathology at FCAV/UNESP, Campus of Jaboticabal, SP, Brazil. The seeds were analyzed by the Blotter test with four repetitions of 50 seeds.
\end{abstract}

\footnotetext{
${ }^{1}$ Submetido em 31/08/2007. Aceito para publicação em 30/04/2008.

${ }^{2}$ Eng. Agr., Doutoranda em Fitotecnia, UFV/DFT, 36570-000, Viçosa, MG, agroneide@hotmail.com;

${ }^{3}$ Eng. Agr., Doutoranda em Agronomia, UFPB/CCA, 58397-000, Areia, PB, leandrabarrozo@hotmail.com.

${ }^{4}$ Eng. Agra., Profa. Assistente Doutor. Depto. de Fitossanidade, Engenharia Rural e Solos, FEIS/UNESP, CP 56, 15385-000, Ilha Solteira, SP, azkronka@agr.feis.unesp.br

${ }^{5}$ Eng. Agrícola, Prof. Assistente Doutor, Dpto. de Engenharia Rural,
}

UNESP/FCAV, Via de Acesso Prof. Paulo Donato Castellane S/N, 14884900, Jaboticabal, SP, rouverson@fcav.unesp.br

${ }^{6}$ Eng. Agra., FESURV, 75.901-970, Rio Verde, GO, apolyana.leao@ hotmail.com

${ }^{7}$ Biologo, Doutorando em Agronomia (PTS) UNESP/FCAV, silvabms@ hotmail.com

${ }^{8}$ Eng. Agra, Profa. Assistente Doutor, Dpto. de Fitossanidade, UNESP/ FCAV, rpanizzi@fcav.unesp.br 
There was significant interaction for the factors machines and collection places for the incidence of Penicillium sp. and Fusarium sp., among machines and speed for Aspergillus spp., and among speed and collection places for Penicillium sp.. The presence of fungi associated to the seeds, mainly, Cladosporium sp. and Fusarium sp. did not depend on the machine type. Smaller contamination in general was verified in the seeds collected from the unloading exit and at the speed of $4 \mathrm{Km} . \mathrm{h}^{-1}$. For Fusarium sp., Aspergillus spp., and Penicillium sp. there was a dependence of the factors on their incidences in the seeds of the cultivars analyzed, under the studied conditions.

Index Terms: Glycine max, mechanical harvesting, mechanical damage, seed pathology

\section{INTRODUÇÃO}

A cultura da soja [Glycine $\max (\mathrm{L}$.) Merrill] ocupa posição de destaque no cenário agrícola nacional, tendo apresentado, nas últimas décadas, uma expansão, não só em termos de área plantada nas regiões tradicionais de cultivo, como também um avanço para novas regiões. Paralelamente à expansão da cultura, surgiu uma série de problemas de ordem fitossanitária, fazendo com que a maioria das doenças de importância econômica também aumentasse, tanto em número quanto em intensidade. Além de problemas de ordem fisiológica, a presença de microorganismos ameaça a qualidade das sementes, principalmente por provocar redução na germinação e por se constituir em uma potencial fonte de inóculo para novas safras.

As danificações provocadas nas sementes por operações mecanizadas realizadas durante a produção podem atuar negativamente na qualidade do material obtido. Essa redução qualitativa, segundo a literatura específica, pode ser agravada quando a injúria interage com microrganismos presentes na superfície das sementes ou no solo, por ocasião da semeadura (Cícero e Silva, 2003). Os danos decorrentes da associação de patógenos com sementes não se limitam apenas a perdas diretas de população de plantas no campo, mas abrangem também uma série de outras implicações que, de forma mais acentuada, podem levar a danos irreparáveis a todo o sistema agrícola (Machado, 1988).

O controle de qualidade de sementes da soja é de fundamental importância dentro do contexto das cadeias produtivas, pois, ou o produtor adota regras claras desse controle, ou provavelmente será eliminado dessa atividade (Costa et al., 2003). Segundo Couto e Alvarenga (1998) a mecanização agrícola é a principal responsável pelos danos mais graves causados aos grãos. Além de apresentarem efeitos imediatos sobre sua qualidade, as predispõem à deterioração mais rápida, pelo aumento da respiração e da lixiviação de eletrólitos, proporcionando um maior percentual no número de plântulas fracas e anormais, maior suscetibilidade a microrganismos, maior sensibilidade aos fungicidas e redução no potencial de armazenamento (Fessel et al., 2003). Segundo Smiderle et al. (2003) o grau dos danos causados pelos patógenos às sementes depende de fatores bióticos, como a intensidade da infecção ou infestação por fungos antes da colheita, de patógenos existentes no solo, e dos danos mecânicos provenientes da colheita, além dos danos após a secagem e beneficiamento, e também das condições do armazenamento.

Diante disso, o objetivo do trabalho foi avaliar a qualidade sanitária de sementes de soja colhidas por duas colhedoras radiais, operando em duas velocidades distintas e provenientes de dois locais de coleta das amostras nas colhedoras.

\section{MATERIAL E MÉTODOS}

Foram utilizadas sementes de soja cultivar Coodetec 217 colhidas na Fazenda Samello III, com $16 \pm 2 \%$ de teor de água, por ocasião da colheita, em abril de 2007, no Município de Sacramento, MG.

Para a colheita da soja, foram operadas duas colhedoras 
Massey Ferguson 5650, Advanced, com rotação de $900 \mathrm{rpm}$, uma ano 2003 e a outra ano 2004, sendo que ambas colhedoras operaram com velocidades de colheita em torno de $4 \mathrm{Km} \cdot \mathrm{h}^{-1}$ e $7 \mathrm{Km} \cdot \mathrm{h}^{-1}$. Amostras de sementes foram coletadas no tanque graneleiro e na saída do tubo de descarga. Foi obtido o teor de água das sementes por meio da secagem de quatro repetições de 100 sementes secas em estufa a $105 \pm 3{ }^{\circ} \mathrm{C}$, durante 24 horas (Brasil, 1992). Em seguida, as sementes de soja foram acondicionadas em papel Kraft e, posteriormente, armazenadas em câmara fria a $10 \pm 3{ }^{\circ} \mathrm{C}$. As análises de sanidade das sementes foram realizadas no Laboratório de Fitopatologia da Faculdade de Ciências Agrárias e Veterinárias da Universidade Estadual Paulista (UNESP/FCAV), Campus de Jaboticabal, SP, Brasil. Foi utilizado o método do papel de filtro em placas de Petri (Blotter test), com quatro repetições de 50 sementes, para a detecção dos fungos. As avaliações foram realizadas examinando-se as sementes, individualmente, com auxílio de microscópio estereoscópico, computandose a porcentagem de sementes, com fungos, em cada tratamento.

Foi empregado o delineamento inteiramente casualizado no esquema fatorial $2 \times 2 \times 2$ ( 2 modelos de colhedora $\times 2$ velocidades $\mathrm{x} 2$ pontos de coleta na máquina). Os dados de porcentagem foram transformados para arc sen $(\mathrm{x} / 100)^{1 / 2}$. Para a análise de variância foi utilizado o teste $\mathrm{F}$ e, para a comparação de médias, o teste de Tukey a $5 \%$ de probabilidade.

\section{RESULTADOS E DISCUSSÕES}

Os teores de água das sementes de soja cv. Coodetec 217 colhidas pelas colhedoras Massey Ferguson 5650, anos 2003 e 2004 são apresentados na Tabela 1.

Os resultados da análise de variância do teste de sanidade das sementes com fungos em geral, indicam, que houve interação significativa entre os fatores colhedora ponto de coleta, e também, entre velocidade e ponto de coleta da colhedora (Tabela 2). Foi verificada a presença dos fungos Fusarium sp., Cercospora kikuchii, Phomopsis sp., Cladosporium sp., Epicoccum sp., Phoma sp. (fungos de campo), Aspergillus spp., Penicillium sp. e Rhizopus sp. (fungos de armazenamento) associados às sementes de soja com índices variáveis entre os tratamentos (Figura 1). Oliveira et al. (2004) realizou um levantamento de fungos em sementes de soja de 14 linhagens desenvolvidas pelo programa de Melhoramento de Soja da Universidade Federal de Goiás e em quatro cultivares comerciais.
Esses também detectaram a incidência de Fusarium sp. e Cercospora kikuchii nas sementes avaliadas.

TABELA 1. Teor de água inicial de sementes de soja cv. Coodetec 217, obtidas no tanque graneleiro e na saída do tubo de descarga para os dois modelos de colhedora Massey Ferguson (MF), operando a 4 km.h ${ }^{-1}$ e 7 km.h. 2007.

\begin{tabular}{|c|c|c|c|}
\hline \multicolumn{3}{|c|}{ Fatores } & $\begin{array}{c}\text { Teor de } \\
\text { água }(\%)\end{array}$ \\
\hline \multirow{4}{*}{$\begin{array}{c}\text { MF } 5650 \\
2003\end{array}$} & \multirow{2}{*}{$4 \mathrm{~km} \cdot \mathrm{h}^{-1}$} & $\begin{array}{c}\text { Tanque } \\
\text { graneleiro }\end{array}$ & 13,5 \\
\hline & & $\begin{array}{c}\text { Saida do tubo de } \\
\text { descarga }\end{array}$ & 12,4 \\
\hline & \multirow{2}{*}{$7 \mathrm{~km} \cdot \mathrm{h}^{-1}$} & $\begin{array}{c}\text { Tanque } \\
\text { graneleiro }\end{array}$ & 11,9 \\
\hline & & $\begin{array}{c}\text { Saida do tubo de } \\
\text { descarga }\end{array}$ & 11,7 \\
\hline \multirow{4}{*}{$\begin{array}{c}\text { MF } 5650 \\
2004\end{array}$} & \multirow{2}{*}{$4 \mathrm{~km} \cdot \mathrm{h}^{-1}$} & $\begin{array}{c}\text { Tanque } \\
\text { graneleiro }\end{array}$ & 11,2 \\
\hline & & $\begin{array}{c}\text { Saida do tubo de } \\
\text { descarga }\end{array}$ & 12,6 \\
\hline & \multirow{2}{*}{$7 \mathrm{~km} \cdot \mathrm{h}^{-1}$} & $\begin{array}{c}\text { Tanque } \\
\text { graneleiro }\end{array}$ & 12,3 \\
\hline & & $\begin{array}{c}\text { Saida do tubo de } \\
\text { descarga }\end{array}$ & 12,7 \\
\hline
\end{tabular}

Pela Tabela 3, observa-se um menor número de sementes com fungos provenientes da saída do tubo de descarga, independente do tipo de colhedora utilizada. Entretanto, na Tabela 4, pode ser observado que houve diferença significativa entre velocidade e ponto de coleta ao se constatar a menor média de sementes com fungos, coletadas na saída do tubo de descarga na velocidade de $4 \mathrm{~km} . \mathrm{h}^{-1}$. Isto demonstra que a menor velocidade pode estar relacionada com o grau de danos mecânicos que acometeram as sementes e que podem ter possibilitado um menor número de sementes com fungos, quando comparado às sementes colhidas na maior velocidade (7 $\mathrm{Km} \cdot \mathrm{h}^{-1}$ ). Segundo Costa et al. (1996), a semente de soja é muito sensível aos impactos mecânicos, uma vez que as partes vitais do embrião, como radículas, hipocótilo e plúmula estão situados sob tegumento pouco espesso, que praticamente não lhes oferece proteção. 
TABELA 2. Síntese da análise de variância referente às médias das sementes de soja cv. Coodetec 217 com fungos em geral, obtidas no tanque graneleiro e na saída do tubo de descarga, para dois modelos de colhedora Massey Ferguson, operando a 4 km.h ${ }^{-1}$ e km.h-1 2007.

Tratamentos

Sementes com fungos

\begin{tabular}{|c|c|}
\hline Colhedoras $(\mathrm{C})$ & \\
\hline Massey Fergunson 2003 & $39,6 \mathrm{a}$ \\
\hline Massey Fergunson 2004 & $37,1 \mathrm{a}$ \\
\hline \multicolumn{2}{|l|}{ Velocidades $(\mathrm{V})$} \\
\hline $4 \mathrm{Km} \cdot \mathrm{h}^{-1}$ & $30,9 \mathrm{~b}$ \\
\hline $7 \mathrm{Km} \cdot \mathrm{h}^{-1}$ & $45,8 \mathrm{a}$ \\
\hline \multicolumn{2}{|l|}{ Local de coleta $(\mathrm{P})$} \\
\hline Tanque graneleiro & $40,6 \mathrm{a}$ \\
\hline Saída do tubo de descarga & $36,1 \mathrm{~b}$ \\
\hline \multicolumn{2}{|c|}{ Teste de F } \\
\hline Colhedora $(\mathrm{C})$ & $2,91^{\mathrm{NS}}$ \\
\hline Velocidade (V) & $108,4^{* *}$ \\
\hline Ponto de coleta $(\mathrm{P})$ & $10,3^{* *}$ \\
\hline Interação CxV & $3,51^{\mathrm{NS}}$ \\
\hline Interação CxP & $4,73^{*}$ \\
\hline Interação VxP & $7,60^{*}$ \\
\hline Interação CxVxP & $0,84^{\mathrm{NS}}$ \\
\hline Desvio padrão & 4,03 \\
\hline $\mathrm{CV}(\%)$ & 10,5 \\
\hline
\end{tabular}

Dados transformados para arc sen $(\mathrm{x} / 100)^{1 / 2}$

NS não significativo; * significativo a $5 \%$; ** significativo a $1 \%$.

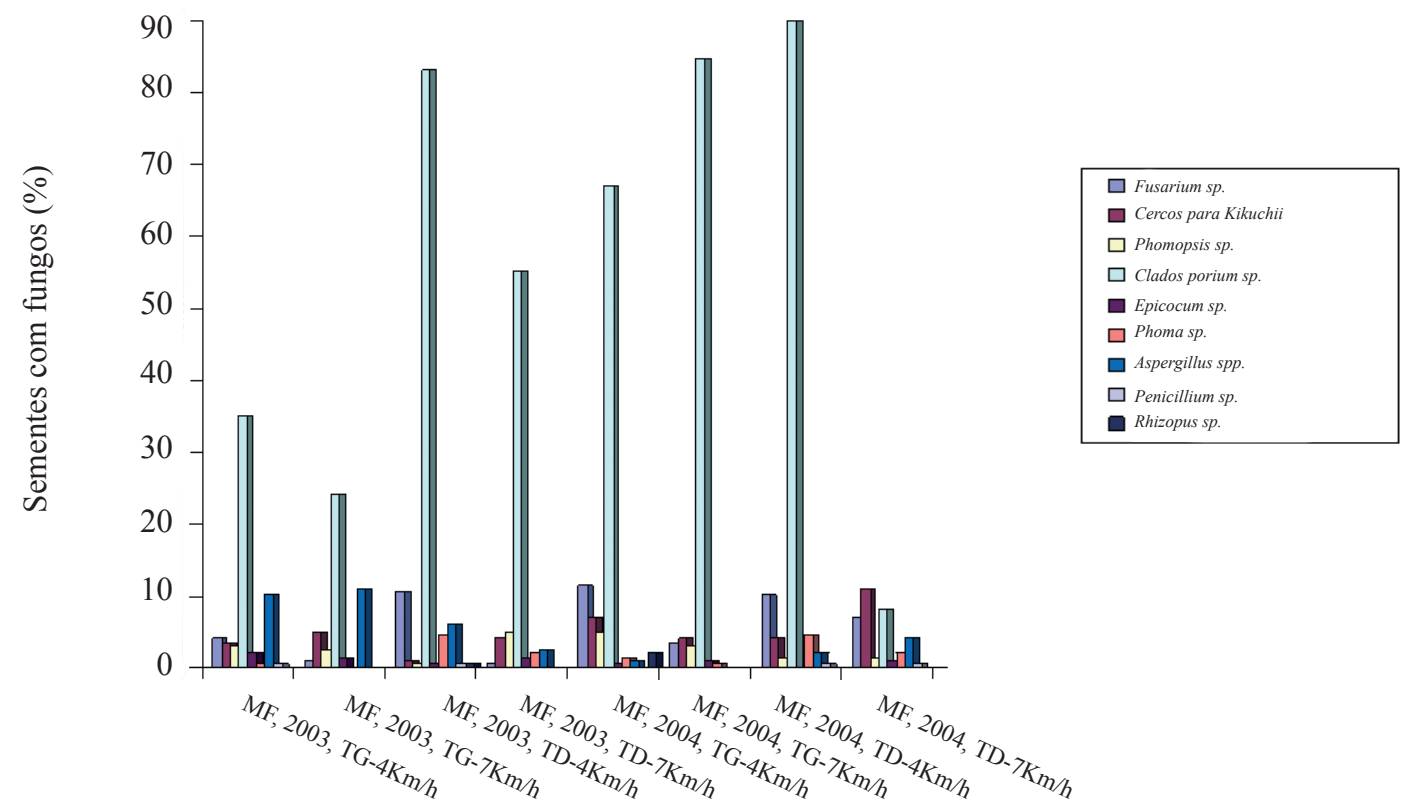

FIGURA 1. Incidência de fungos em sementes de soja cv. Coodetec 217 obtidas no tanque graneleiro (TG) e na saída do tubo de descarga (TD) para dois modelos de colhedora Massey Ferguson (MF), operando a $4 \mathrm{~km} \mathrm{~h}^{-1} \mathrm{e}$ $7 \mathrm{~km} \mathrm{~h}^{-1} .2007$. 
TABELA 3. Incidência geral de fungos em sementes de soja cv. Coodetec 217 colhidas por diferentes colhedores e coletados em pontos absolutos.

\begin{tabular}{ccc}
\hline \multirow{2}{*}{ Colhedora } & \multicolumn{2}{c}{ Ponto de Coleta } \\
\cline { 2 - 3 } & $\begin{array}{c}\text { Tanque } \\
\text { graneleiro }\end{array}$ & $\begin{array}{c}\text { Saída do tubo de } \\
\text { descarga }\end{array}$ \\
\hline Massey Ferguson 2003 & $43,4 \mathrm{Aa}$ & $35,7 \mathrm{Ba}$ \\
Massey Ferguson 2004 & $37,9 \mathrm{Ab}$ & $36,4 \mathrm{Aa}$ \\
\hline $\mathrm{dms}$ & \multicolumn{2}{c}{4,16} \\
\hline
\end{tabular}

Dados transformados para arc sen arc sen $(\mathrm{x} / 100)^{1 / 2}$

Médias seguidas de mesma letra minúscula, na coluna,não diferem pelo teste de Tukey $(\mathrm{P}>0,05)$.

Médias seguidas de mesma letra maiúscula, na linha, não diferem pelo teste de Tukey $(\mathrm{P}>0,05)$.

Na Tabela 5, observam-se os resultados da análise de variância do teste de sanidade das sementes de soja com Fusarium sp., Phomopsis sp., Cercospora sp., Aspergillus spp. e Penicillium sp.. Houve interação significativa para os fatores colhedora e ponto de coleta para as médias de sementes de soja com os fungos Penicillium sp. e Fusarium
TABELA 4. Incidência geral de fungos em sementes de soja cv. Coodetec 217 colhidas com diferentes velocidades e coletados em pontos distintos.

\begin{tabular}{ccc}
\multirow{2}{*}{ Velocidade } & \multicolumn{2}{c}{ Ponto de Coleta } \\
\cline { 2 - 3 } & Tanque graneleiro $\begin{array}{c}\text { Saída do tubo de } \\
\text { descarga }\end{array}$ \\
\hline $4 \mathrm{Km} \cdot \mathrm{h}^{-1}$ & $35,2 \mathrm{Ab}$ & $26,6 \mathrm{Bb}$ \\
$7 \mathrm{Km} \cdot \mathrm{h}^{-1}$ & $46,1 \mathrm{Aa}$ & $45,5 \mathrm{Aa}$ \\
\hline $\mathrm{dms}$ & \multicolumn{3}{|c}{4,16} \\
\hline
\end{tabular}

Dados transformados para arc sen $\operatorname{arc} \operatorname{sen}(\mathrm{x} / 100)^{1 / 2}$

Médias seguidas de mesma letra minúscula, na coluna,não diferem pelo teste de Tukey $(\mathrm{P}>0,05)$.

Médias seguidas de mesma letra maiúscula, na linha, não diferem pelo teste de Tukey $(\mathrm{P}>0,05)$.

sp.; entre colhedora e velocidade para Aspergillus spp.; e também, entre velocidade e ponto de coleta, para as médias de sementes com Penicillium sp., sendo que para a contaminação de sementes com esse fungo, houve a interação significativa entre os três fatores. Os desdobramentos dessas interações estão nas Tabelas 6 e 7.

TABELA 5. Análise de variância para a porcentagem de sementes de soja cv. Coodetec 217 com fungos considerando os fatores colhedora, velocidade e ponto de colheita.

\begin{tabular}{|c|c|c|c|c|c|}
\hline FATORES & Phomopsis sp. & Penicillium sp. & Fusarium sp. & C.kikuchii, & Aspergillus spp. \\
\hline \multicolumn{6}{|l|}{ Colhedoras (C) } \\
\hline $\mathrm{C} 1=\mathrm{MF} 2003$ & $6,14 \mathrm{a}$ & $4,00^{(1)}$ & 12,12 & $10,65 \mathrm{a}$ & 9,81 \\
\hline $\mathrm{C} 2=\mathrm{MF} 2004$ & $7,20 \mathrm{a}$ & 0,89 & 10,44 & $11,27 \mathrm{a}$ & 10,76 \\
\hline \multicolumn{6}{|l|}{ Velocidades $(\mathrm{V})$} \\
\hline $\mathrm{V} 1=4 \mathrm{Km} \cdot \mathrm{h}^{-1}$ & $8,45 \mathrm{a}$ & 3,03 & $2,92 \mathrm{~b}$ & $13,22 \mathrm{a}$ & 13,55 \\
\hline $\mathrm{V} 2=7 \mathrm{Km} \cdot \mathrm{h}^{-1}$ & $4,89 \mathrm{a}$ & 1,85 & $15,34 \mathrm{a}$ & $8,70 \mathrm{a}$ & 7,01 \\
\hline \multicolumn{6}{|l|}{ Pontos de coleta $(\mathrm{P})$} \\
\hline $\mathrm{P} 1=$ Tanque graneleiro & $8,45 \mathrm{a}$ & 3,03 & 11,71 & $10,26 \mathrm{a}$ & $10,01 \mathrm{a}$ \\
\hline $\mathrm{P} 2=$ Tubo de descarga & $4,89 \mathrm{a}$ & 1,85 & 10,86 & $11,66 \mathrm{a}$ & $10,56 \mathrm{a}$ \\
\hline \multicolumn{6}{|l|}{ Teste F } \\
\hline Colhedora $(\mathrm{C})$ & $14,71^{\mathrm{NS}}$ & $11,76 * *$ & $0,63^{\mathrm{NS}}$ & $0,07^{\mathrm{NS}}$ & $0,22^{\mathrm{NS}}$ \\
\hline Velocidade (V) & $0,27^{\mathrm{NS}}$ & $1,69^{\mathrm{NS}}$ & $16,81 * *$ & $3,73^{\mathrm{NS}}$ & $10,27 * *$ \\
\hline Ponto de coleta $(\mathrm{P})$ & $13,04^{\mathrm{NS}}$ & $1,69^{\mathrm{NS}}$ & $0,16^{\mathrm{NS}}$ & $0,36^{\mathrm{NS}}$ & $0,07^{\mathrm{NS}}$ \\
\hline Interação C x V & $28,80^{\mathrm{NS}}$ & $0,06^{\mathrm{NS}}$ & $1,50^{\mathrm{NS}}$ & $1,54^{\mathrm{NS}}$ & $25,42 * *$ \\
\hline Interação C x P & $0,32^{\mathrm{NS}}$ & $5,59^{\mathrm{NS}}$ & $9,74 * *$ & $0,04^{\mathrm{NS}}$ & $1,32^{\mathrm{NS}}$ \\
\hline Interação V x P & $11,89^{\mathrm{NS}}$ & $5,59^{*}$ & $3,17^{\mathrm{NS}}$ & $4,15^{\mathrm{NS}}$ & $0,19^{\mathrm{NS}}$ \\
\hline Interação C x V x P & $4,97^{\mathrm{NS}}$ & $11,76^{* *}$ & $1,41^{\mathrm{NS}}$ & $1,14^{\mathrm{NS}}$ & $0,33^{\mathrm{NS}}$ \\
\hline Desvio padrão & 5,31 & 2,56 & 6,01 & 6,60 & 5,78 \\
\hline
\end{tabular}

$\mathrm{a}, \mathrm{b}$ - em cada coluna, para cada fator, médias seguidas de mesma letra não diferem $(\mathrm{P}>0,05)$

NS - não significativo $(\mathrm{P}>0,05) \quad *$ - significativo $(\mathrm{P}<0,05) \quad * *$ - significativo $(\mathrm{P}<0,01)$

(1) - médias não seguidas por letras estão envolvidas em interação significativa 
TABELA 6 . Incidência de Penicillium sp. e Fusarium sp. em sementes de soja cv. Coodetec 217 colhidas por diferentes colhedoras (C), e velocidades $(\mathrm{V})$ e coletados em pontos de coleta (P) distintos.

\begin{tabular}{ccc}
\hline Combinações & Penicillium sp. & Fusarium $\mathrm{sp}$. \\
\hline C1P1 & 2,34 b A & 9,23 a A \\
C1P2 & 5,66 a A & 15,02 a A \\
C2P1 & 1,37 a A & 14,19 a A \\
C2P2 & 0,41 a B & 6,70 b B \\
\hline
\end{tabular}

$\mathrm{a}, \mathrm{b}$ - em cada coluna, médias de Pontos de Coleta em cada Colhedora seguidas de mesma letra minúscula não diferem $(\mathrm{P}>0,05)$.

A, B - em cada coluna, médias de Colhedoras em cada Ponto de Coleta seguidas de mesma letra maiúscula não diferem $(\mathrm{P}>0,05)$.

TABELA 7. Incidência de Aspergillus spp., em diferentes Colhedoras (C) $x$ Velocidades (V), e Velocidades (V) e Pontos de Coleta (P) para a incidência de Penicillium $\mathrm{sp}$. em sementes de soja cv. Coodetec 217.

\begin{tabular}{|c|c|c|c|}
\hline \multicolumn{4}{|c|}{ Combinações Aspegillus spp Combinações Penicillium sp } \\
\hline C1V1 & $35,01 \mathrm{~b} \mathrm{~B}$ & V1P1 & 9,83 b B \\
\hline $\mathrm{C} 1 \mathrm{~V} 2$ & $35,79 \mathrm{~b} \mathrm{~A}$ & V1P2 & 22,15 a $\mathrm{A}$ \\
\hline $\mathrm{C} 2 \mathrm{~V} 1$ & 45,80 a $A$ & V2P1 & 17,63 a $\mathrm{A}$ \\
\hline $\mathrm{C} 2 \mathrm{~V} 2$ & $29,70 \mathrm{~b} \mathrm{~B}$ & $\mathrm{~V} 2 \mathrm{P} 2$ & 15,83 a B \\
\hline
\end{tabular}

Para a Interação C x V:

a, $\mathrm{b}$ - médias de Velocidades, em cada Colhedora, seguidas de mesma letra minúscula não diferem $(\mathrm{P}>0,05)$

A, B - médias de Colhedoras, em cada Velocidade, seguidas de mesma letra maiúscula não diferem $(\mathrm{P}>0,05)$

Para a Interação V x P:

$\mathrm{a}, \mathrm{b}$ - médias de Pontos de Coleta em cada Velocidade seguidas de mesma letra minúscula não diferem $(\mathrm{P}>0,05)$

A, B - médias de Velocidades em cada Ponto de Coleta seguidas de mesma letra maiúscula não diferem $(\mathrm{P}>0,05)$.

De acordo com a Tabela 6, verifica-se que, para a incidência de Penicillium sp. nas sementes de soja provenientes da colhedora MF 2003, houve diferença para os dois pontos de coleta; entretanto, somente para o ponto do tubo de descarga observa-se diferença entre as colhedoras. Já para o fungo Fusarium sp., observa-se que não houve diferença entres as médias obtidas para as sementes coletadas no tanque graneleiro, em ambas as colhedoras, e nem entre os pontos de coleta para a colhedora MF 2003.

$\mathrm{Na}$ interação significativa entre colhedora e velocidade de colheita, para as médias de sementes de soja com Aspergillus spp. (Tabela 7), observa-se, para ambas as velocidades, que houve diferença entre as colhedoras; entretanto, só houve diferença entre as velocidades para a colhedora MF 2004. Deve-se destacar, que numericamente, uma maior média de sementes com Aspergillus spp., é verificada quando essas foram colhidas na saída do tubo de descarga pela colhedora MF 2004. Com respeito à interação entre velocidade e ponto de coleta, para as médias de sementes com Penicillium sp., em ambos os pontos de coleta houve diferença entre as velocidades, e somente, na velocidade de $4 \mathrm{~km} . \mathrm{h}^{-1}$ ocorreu diferença entre os pontos de coleta.

Segundo Oliveira et al. (1997), para que os fungos possam causar algum dano às sementes, esses terão que invadir e colonizar os tecidos internos das mesmas, sendo favorecidos pelas condições de alta umidade e alta temperatura, e principalmente, pela presença de danos mecânicos na camada protetora das sementes. Em geral, as sementes aqui analisadas demonstraram adequado teor de água (Tabela 1), o que favorece a hipótese de que o dano mecânico por trincagem ou quebra, e não o teor de água das sementes foi o fator preponderante que contribuiu para a infecção fúngica. Para Carvalho \& Nakagawa (2000), as danificações mecânicas são conseqüências de regulagens não adequadas das máquinas ou equipamentos que realizam operações de colheita e beneficiamento das sementes, principalmente quando essas apresentam alto ou baixo teor de água. Segundo esses autores, a quebra de sementes, danificação comum e que também foi presente nas sementes desse experimento, é mais intensa entre 12 a $14 \%$ de água na semente, teores estes, também, observados nesta pesquisa, sendo que esse dano pode atingir diferentes partes da semente, comprometendo assim, um determinado lote.

De acordo com Machado (1988), os danos provocados pelos patógenos, podem estar diretamente relacionados com o potencial de inóculo e com sua localização na semente. Isso nos remete a afirmar que, mesmo havendo incidência alta ou baixa dos fungos estudados nestas sementes, uma preocupação maior deve estar mais relacionada não tão somente a essa incidência, mas também à sua localização na semente, principalmente, se esta for localizada próximo ao embrião. E mesmo que estes fungos não venham a causar danos imediatos, existem os latentes, os quais são cumulativos durante o armazenamento, que quando não controlado, poderá, consequentemente, desencadear a deterioração das sementes, pelo fato desses danos serem a porta de entrada 
para diferentes espécies de Aspergillus e Penicillium, que se desenvolvem em condições de alta temperatura e umidade relativa do ar, mas também são capazes de sobreviver em ambientes com baixa umidade, proliferando em sucessão aos fungos de campo e causando a deterioração das sementes (Wetzel, 1987).

\section{CONCLUSÕES}

A presença de fungos associados às sementes de soja cv. Coodetec 217, principalmente, Cladosporium sp. e Fusarium sp., independe do tipo de colhedora utilizada.

Menor contaminação fúngica em geral, verifica-se nas sementes coletadas na saída do tubo de descarga à $4 \mathrm{Km} \cdot \mathrm{h}^{-1}$.

Os fatores estudados não interagem para uma incidência expressiva de Cercospora kikuchii e Phomopsis sp. nas sementes analisadas, enquanto que para Fusarium sp., Aspergillus spp., e Penicillium sp. existe uma dependência variável dos fatores sobre suas incidências na condição experimental observada.

\section{REFERÊNCIAS}

BRASIL. Ministério da Agricultura e Reforma Agrária. Secretaria Nacional de Defesa Agropecuária. Departamento Nacional de Produção Vegetal. Coordenação de Laboratório Vegetal. Regras para Análise de Sementes. 1992. 365 p.

CARVALHO, N.M.; NAKAGAWA, J. Sementes: ciência, tecnologia e produção. 4. ed. Jaboticabal: FUNEP, 2000. $588 \mathrm{p}$.

CICERO, C. M.; SILVA, W. R. da. Danos mecânicos associados a patógenos e desempenho de sementes de milho. Bragantia, v.62, n.2, p.305-314, 2003.

COSTA, N. P. da.; MESQUITA, C. de M.; MAURINA, A. C.; FRANÇA NETO, J. de B.; KRZYZANOWSKI, F. C.;
HENNING, A. A. Qualidade fisiológica, física e sanitária de sementes de soja produzidas no Brasil. Revista Brasileira de Sementes, v.25, n.1, p.128-132, 2003.

COSTA, N.P.; OLIVEIRA, M.C.N.; HENNING, A.A.; KRZYZANOWSKI, F.C.; MESQUITA, C.M.; TAVARES, L.C.V. Efeito da colheita mecânica sobre a qualidade da semente de soja. Revista Brasileira de Sementes, v.18, p.232-237, 1996.

COUTO, S. M.; ALVARENGA, L. C. Resistência de grãos de soja a impactos mecânicos. Revista Brasileira de Armazenagem, v. 23, n. 2, p. 3-9, 1998.

FESSEL, S.A.; SADER, R.; DE PAULA, R.D.; GALLI, J.A. Avaliação da qualidade física, fisiológica e sanitária de sementes de milho durante o beneficiamento. Revista Brasileira de Sementes, v.25, n.2, p.70-76, 2003.

MACHADO, J. da C. Patologia de sementes. fundamentos e aplicação. Brasília, DF: MEC/ Lavras: ESAL/FAEPE, $1988,107 \mathrm{p}$.

OLIVEIRA, E. de.; PINHEIRO, J.B.; ROSSETO, C.A.V. Qualidade fisiológica e sanitária de sementes de genótipos de soja (Glycine max (L.) Merril) cultivadas em diferentes ambientes do Estado de Goiás. Fitopatologia Brasileira, 2004, v. 29, Suplemento, p.129.

OLIVEIRA, J.A.; CARVALHO, M.L.M.de.; PINHO, E.V.R. V. Efeito do método de colheita na qualidade física, fisiológica e sanitária de sementes de milho. Revista Brasileira de Sementes, v.19, n.2, p.200-206, 1997.

SMIDERLE, O. J.; GIANLUPPI, D.; MOURÃO JUNIOR, M.; Tratamento e qualidade de sementes de milho durante o armazenamento em Roraima. Revista Acadêmica: ciências agrárias e ambientais, v.1, n.4, p. 75-83, 2003.

WETZEL, M.V. da S. Fungos de armazenamento. In: SOAVE, J.; WETZEL, M.M.V.S. (Ed.). Patologia de sementes. Campinas: Fundação Cargill, 1987, cap. 12, p. 260-274. 